\title{
25 Years of the Laboratory of Chemical Reaction Engineering at the Swiss Federal Institute of Technology in Lausanne
}

The Laboratory of Chemical Reaction Engineering was founded in 1977 as the third laboratory of the Institute of Chemical Engineering at the EPFL to provide teaching and research in this important domain of chemical engineering science. After 25 years, we take the opportunity to present our current research activities in this issue of CHIMIA.

Chemical reaction engineering centers on the design and control of chemical reactors for the transformation of primary materials into final products. In order to make the transformation processes sustainable, the following requirements must be fulfilled:

- Efficient use of raw material and energy

- Minimal waste production

- Inherently safe reactor operation

To reach these targets, an integrated approach based on chemistry, physics, mathematics and engineering sciences is required. Therefore, we find our laboratory well placed within the new Faculty of Basic Science created at the beginning of 2002. This should facilitate an interdisciplinary interaction in research and teaching activities needed for effective work in the scientific area of chemical reaction engineering.

Fundamental research in chemical kinetics and transport phenomena is a basic activity in the laboratory. Understanding primary chemical and physical processes enables innovative solutions in reactor design and operation to be developed. Two examples of our work in past years are: (1) application of forced sustained unsteady state of catalyst for improved selectivity and enhanced reactor performance and (2) design of advanced polymerization reactors based on the quantitative relationships between micro-mixing and chemical reaction kinetics.

Our current research work is oriented towards the development of new approaches in chemical reaction engineering such as: (i) the design of multi-structured devices for improved process efficiency, (ii) the use of electrochemical catalyst activation for increased activity, (iii) the application of supercritical fluids as reaction media, and (iv) novel tools for the design of inherently safe chemical processes. The articles which follow give an overview of the ongoing research projects in chemical reaction engineering. We are grateful to Prof. J.C. Charpentier, the president of the European Federation for Chemical Engineering, and to Dr. P. Larpin, Head of Technical Operations, Ciba Specialty Chemicals, and coworkers for their contributions presenting an overview in chemical engineering research, educational set-up and industrial application.

Prof. Dr. A. Renken
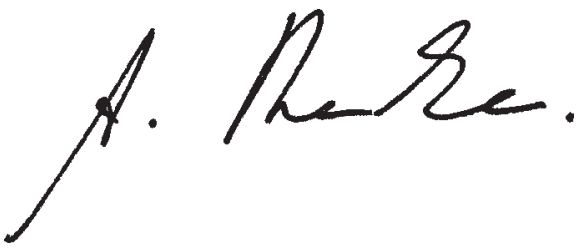

We would also like to dedicate this issue to the 60th birthday of Prof. A. Renken. Everyone who knows him will surely join me in wishing him a 'Happy Birthday'. During the past 25 years, $55 \mathrm{PhD}$ students have graduated from his laboratory and more than 120 diploma projects have been completed, indicating the relevance of chemical reaction engineering. The future will be the creation of a research center on reaction engineering for which Prof. A. Renken is acting as the 'catalyst'.

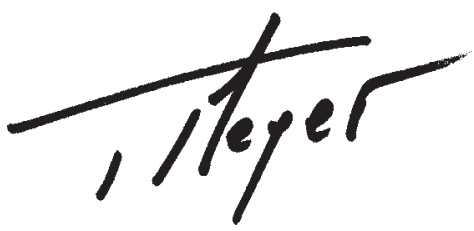

